\title{
Virus-like particles of recombinant PCV2b carrying FMDV-VP1 epitopes induce both anti-PCV and anti-FMDV antibody responses
}

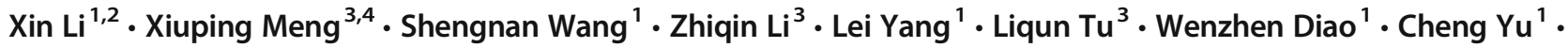 \\ Yongli $\mathrm{Yu}^{3} \cdot$ Chaoying $\mathrm{Yan}^{1,5} \cdot$ Liying Wang ${ }^{1}$
}

Received: 15 July 2018 / Revised: 19 August 2018 / Accepted: 1 September 2018 / Published online: 18 October 2018

(C) Springer-Verlag GmbH Germany, part of Springer Nature 2018

\begin{abstract}
Mixed infection of porcine circovirus type 2 (PCV2) and foot-and-mouth disease virus (FMDV) is devastating to swine populations. To develop an effective vaccine that can protect the pigs from the infection of PCV2 and FMDV, we used the neutralizing B cell epitope region (aa 135-160) of FMDV to replace the regions aa 123-151 and aa 169-194 of the PCV2b Cap protein to generate a recombinant protein designated as Capfb. The Capfb protein was expressed in Escherichia coli system and the purified Capfb protein assembled into virus-like particles (VLPs) through dialysis. The ability of the Capfb protein to induce effective immune response against FMDV and PCV2b was tested in mice and guinea pigs. The results showed that the Capfb-VLPs could elicit anti-PCV2b and anti-FMDV antibody response in mice and guinea pigs without inducing antibodies against decoy epitope. Moreover, the Capfb-VLPs could enhance the percentage and activation of B cells in lymph nodes when the mice were stimulated with inactivated FMDV or PCV2b. These data suggested that the Capfb-VLPs could be an efficacious candidate antigen for developing a novel PCV2b-FMDV bivalent vaccine.
\end{abstract}

Keywords Porcine circovirus type $2 \cdot$ Foot-and-mouth disease virus $\cdot$ Vaccine $\cdot$ Virus-like particle $\cdot$ Antibody response

Electronic supplementary material The online version of this article (https://doi.org/10.1007/s00253-018-9361-2) contains supplementary material, which is available to authorized users.

Chaoying Yan

yanchaoying224@126.com

Liying Wang

wlying@jlu.edu.cn

1 Department of Molecular Biology in College of Basic Medical Sciences and Institute of Pediatrics in The First Hospital of Jilin University, Norman Bethune Health Science Center, Jilin University, Changchun 130021, Jilin, China

2 Department of Pathophysiology, School of Basic Medical Sciences, Zhengzhou University, Zhengzhou 450001, China

3 Department of Immunology, College of Basic Medical Sciences, Norman Bethune Health Science Center, Jilin University, Changchun 130021, Jilin, China

4 Department of Endodontics, School and Hospital of Stomatology, Jilin University, 1500 Qinghua Road, Changchun 130021, China

5 Department of Pediatrics, The First Hospital of Jilin University, No. 71 Xinmin Street, Changchun 130021, China

\section{Introduction}

Porcine circovirus type 2 (PCV2), a member of the Circovirus genus of the Circoviridae, is a small non-enveloped virus containing a circular single-stranded DNA genome with 1.76-kb length (Huang et al. 2011), which mainly encodes replicase protein and capsid (Cap) protein. The Cap protein encoded by open reading frame 2 (ORF2) can independently assemble into virus-like particles (VLPs) and is major immunogen for vaccine development (Mankertz et al. 2004; Nawagitgul et al. 2000). PCV2 is considered to be one of the most important pathogens essentially in all major swineproducing countries (Gillespie et al. 2009), since it could cause PCV-associated disease (PCVAD) which includes wasting, increased mortality, respiratory signs, enteritis, reproductive failure, and porcine dermatitis and nephropathy syndrome (PDNS) in pigs (Opriessnig et al. 2007). Moreover, immunosuppression triggered by PCV2 could make infected animals susceptible to other pathogens (Allan et al. 2004) usually resulting in mixed infections. However, the commercial PCV2 
vaccines could only prevent the pigs from PCV2 (Beach and Meng 2012) instead of the mixed infection.

Foot-and-mouth disease virus (FMDV) is one of the most devastating viruses to swine populations when the pigs are coinfected with PCV2 (Allan et al. 2004). It is highly infectious and could cause foot-and-mouth disease (FMD) in over 70 cloven-hoofed species such as cattle, sheep, and swine (Grubman and Baxt 2004; Stenfeldt et al. 2014). Infected animals exhibit a high fever, followed by blister-like sores in the mouth and on the feet resulting in lameness and increased death rate (Alexandersen et al. 2003). FMDV is a singlestranded, positive-sense RNA virus belonging to Aphthovirus genus and contains a capsid composed of four structural proteins identified as VP1, VP2, VP3, and VP4 of FMDV (Doel and Collen 1982). Among these proteins, VP1 can induce neutralizing antibodies in hosts through the immunogenic site located in the G-H loop (Saiz et al. 2002). The G$\mathrm{H}$ loop is a protruding element on the surface of the FMDV capsid spanning about 20 residues around positions 140-160 (Kupper et al. 1981; Pfaff et al. 1982) and contains a highly conserve Arg-Gly-Asp (RGD) motif which interacts with cell surface integrin receptors and contributes to the induction of neutralizing antibodies (Jackson et al. 2002; Verdaguer et al. 1996). A synthetic peptide comprising amino acid (aa) 140160 of the type O FMDV-VP1 protein could induce the production of neutralizing antibodies in guinea pigs (Bittle et al. 1982). Another recombinant vaccine involving aa $141-160$ of the type O FMDV-VP1 protein and the swine immunoglobulin $\mathrm{G}$ heavy-chain constant region against type O FMDV could also induce high level of anti-FMDV-specific antibodies in swine and provide protection (Shao et al. 2011). Thus the G-H loop is an important immunogenic region for developing novel vaccines against FMDV.

The subunit vaccine based on VLPs is not only effective to stimulate immune response but also non-infectious and can be easily produced on a large scale (Crisci et al. 2012; Zeltins 2013). Therefore, many types of VLPs have been tried as carriers for foreign epitopes to induce antibody responses against target antigens. VLPs formed by VP2 of porcine parvovirus (PPV) carrying $\mathrm{CD}^{+} \mathrm{T}$ cell epitope of the lymphocytic choriomeningitis virus (LCMV) in the $\mathrm{N}$-terminus can induce strong responses of cytotoxic $\mathrm{T}$ lymphocyte (CTL) which could kill virally infected cells, and confer total protection against LCMV challenge in mice (Andersen et al. 2006) (Sedlik et al. 1997). VLPs formed by PCV2 have also been successfully used as foreign epitope carriers (Zhang et al. 2014). Chimeric VLPs of PCV2 generated by replacing the nuclear localization signal (NLS) of PCV2 Cap protein with classical swine fever virus (CSFV) T cell epitope and B cell epitope could form VLPs and induce efficient humoral and cellular immunity against PCV2 and CSFV in mice (Zhang et al. 2014). However, the decoy epitope (aa 169-180) recently identified in the Cap of PCV2 has been demonstrated to divert the antibody response away from protective epitopes (Trible et al. 2012). The decoy epitope forms an external loop structure protruding from the surface in the Cap monomer, while it is buried near the interface of the 3 -fold axis in the icosahedral PCV2 particles (Trible et al. 2012). Therefore, the antibodies induced by this epitope display low neutralizing activity and could not confer protection. Up to now, four major genotypes of PCV2 have been identified: PCV2a, PCV2b, PCV2c, and PCV2d, and among them, PCV2b is the most widespread genotype in recent years (Franzo et al. 2016).

Thus, to develop an effective vaccine that can protect the pigs from the mixed infection of PCV2 and FMDV, the neutralizing B cell epitope region (aa 135-160) containing G-H loop of type O FMDV-VP1 protein was used to replace the region (aa 169-194) involving the decoy epitope (aa 169180 ) and a protruding region (aa 123-151) of the PCV2b Cap protein to generate a recombinant protein designated as Capfb. The Capfb protein expressed in the Escherichia coli system was assembled into VLPs in vitro and its efficacy was subsequently evaluated in mice and guinea pigs.

\section{Materials and methods}

\section{Protein structure prediction}

The secondary and 3D structures of the recombinant protein were predicted by DNASTA Protean software and the Swiss Model website (http://swissmodel.expasy.org/), respectively. The PyMOL software was used to visualize3D structures.

\section{Construction of recombinant expression plasmid}

The recombinant pET28a plasmid encoding Cap protein of PCV2b with sixhistidine tag (pET28a-CP-His) was constructed in our lab as previously described (Yu et al. 2016). Using the pET28a-CP-His as a template, fragments A, B, and C were amplified by PCR with the primers shown in Table 1 (Fig. $\mathrm{S} 1 \mathrm{~A})$. Then the fragments $\mathrm{A}$ and $\mathrm{B}$ were cloned into pET28a plasmid with the modified multiple cloning sites using $N c o$ I and EcoRI and EcoRI and BamHI, respectively (Fig. S1B). Finally, the resultant plasmid digested with Bam HI and $\mathrm{XhoI}$ was ligated with the fragment $\mathrm{C}$ digested with $B g l I I$ and $X h o I$ sites to obtain the pET28a-Capfb-His plasmid which could encode the Capfb protein (Fig. S1B).

\section{Expression and purification of recombinant protein}

The pET28a-Capfb-His were transformed into E. coli BL21 (DE3) (Invitrogen) for expressing the Capfb protein under IPTG induction. Then the Capfb protein was purified by nickel affinity chromatography (GE Healthcare, UK). The supernatant from the lysate of the $E$. coli was loaded onto the nickel 
Table 1 Primers used in this study

\begin{tabular}{|c|c|c|c|}
\hline Genes & Names & Primer sequence $\left(5^{\prime}-3^{\prime}\right)$ & Restriction site \\
\hline \multirow[t]{2}{*}{ Fragment A } & $1 \mathrm{~F}$ & CCATGGGTATGACTTATCCACGTCG & NcoI \\
\hline & $1 \mathrm{R}$ & GAATTCGACCTTCACCTTGCGGATG & EcoRI \\
\hline \multirow[t]{4}{*}{ Fragment B } & $1 \mathrm{~F}$ & CTCCAAATACGCTGAAGGTTCTCTGACCAACGTCCGTGGTGACCTTCAAGTATTGGCTC & \\
\hline & $1 \mathrm{R}$ & GGTAAGAAAACGGCTGCGGAATTGGAAGTGGACGAGCAGCCTTCTGAGCCAATACTTGA & \\
\hline & $2 \mathrm{~F}$ & GAATTCTGGCCATGTTCTCCTATTACTCAAGGTGATCGTGGTGTCGGCTCCAAATACGC & EcoRI \\
\hline & $2 \mathrm{R}$ & GGATCCTTCAGCGTATTTGACTGGTTTCGGAGTGAAATAACGGGAGTGGTAAGAAAACG & BamHI \\
\hline \multirow[t]{4}{*}{ Fragment $\mathrm{C}$} & $1 \mathrm{~F}$ & AAGTATTGGCTCAGAAGGCTGCTCGTCCACTTCCAGTTGATCACGTCGGACTT & \\
\hline & $1 \mathrm{R}$ & CTCGAGCTATTAGTGGTGGT & XhoI \\
\hline & $2 \mathrm{~F}$ & AGATCTCTGACCAACGTCCGTGGTGACCTTCAAGTATTGGCTCAGAAG & $B g l I I$ \\
\hline & $2 \mathrm{R}$ & CTCGAGCTATTAGTGGTGGT & XhoI \\
\hline
\end{tabular}

The sequences in bold represent the restriction sites

$F$ forward primer, $R$ reverse primer

affinity column. Unbound proteins were removed by washing the column with washing buffer (20 mM Tris, $500 \mathrm{mM} \mathrm{NaCl}$, $30 \mathrm{mM}$ imidazole, $10 \mathrm{mMb}$-mercaptoethanol, $5 \%$ glycerol, $0.05 \%$ Tween 80, $\mathrm{pH} 8.0$ ) and the bound Capfb protein was eluted in an elution buffer $(20 \mathrm{mM}$ Tris, $500 \mathrm{mM} \mathrm{NaCl}$, $500 \mathrm{mM}$ imidazole, $50 \mathrm{mM}$ b-mercaptoethanol, $5 \%$ glycerol, $0.05 \%$ Tween 80, $\mathrm{pH} 8.0$ ). The Capfb protein expressed in E. coli and purified by nickel affinity chromatography was analyzed by sodium dodecyl sulfate polyacrylamide gel electrophoresis (SDS-PAGE).

\section{Western blot}

The E. coli lysates were subjected to $12 \%$ SDS-PAGE and then transferred onto nitrocellulose membrane (Whatman, Germany) using TE 77 PWR Semi-Dry Transfer Unit (GE Healthcare, UK). Specific antibody raised from ICR mice immunized with inactivated PCV2b or FMDV (1:100 dilution) were used to identify the Capfb protein.

\section{Transmission electron microscopy}

The purified Capfb protein was dialyzed against assembly buffer $\left(50 \mathrm{mM} \mathrm{Na}_{2} \mathrm{HPO}_{4}, 50 \mathrm{mM} \mathrm{NaH}{ }_{2} \mathrm{PO}_{4}, 500 \mathrm{mM} \mathrm{NaCl}\right.$, $5 \%$ glycerol, $0.03 \%$ Tween $80,1 \%$ Triton $\mathrm{X}-100, \mathrm{pH} 6.5$ ) to yield assembled VLPs. The VLPs were observed under transmission electron microscopy (TEM) (FEI, Tecnai Spirit, the Netherlands) at an accelerating voltage of $80 \mathrm{kV}$ and photographed at a magnification of 40,000 . The Cap protein of PCV2b expressed and assembled as previously described (Yu et al. 2016) was used as a control.

\section{Vaccine preparation}

The Capfb-VLPs or the Cap-VLPs were formulated with ISA 35 adjuvant, an oil-based adjuvant of oil-in-water $(\mathrm{O} / \mathrm{W})$ emulsion (Seppic $®$, France), in a 3:1 volume ratio (antigen volume:adjuvant volume) for preparing Capfb or Cap vaccine.

\section{Animals and immunizations}

Animal experiments were performed according to the protocols approved by the Scientific Investigation Board of Science \& Technology of Jilin Province and in accordance with the National Institute of Health Guide for the Care. Specific pathogen-free female ICR mice $(20 \pm 1 \mathrm{~g})$ and female guinea pigs (about $300 \mathrm{~g}$ ) were obtained from the Experimental Animal Center, Medical College of Norman Bethune, Jilin University.

ICR mice, six in each group, were intramuscularly immunized with Capfb or Cap vaccine (10 $\mu \mathrm{g} /$ mouse) on days 0 and 14, and bled on days 0,28 , and 35 for isolating serum. Guinea pigs, four in each group, were intramuscularly immunized with Capfb or Cap vaccine $(20 \mu \mathrm{g} / \mathrm{guinea}$ pig) on days 0,14 , and 28 , and bled on days 0 and 42 for isolating serum.

\section{Enzyme-linked immunosorbent assay}

Inactivated FMDV or PCV2b was used to detect the FMDVspecific or PCV2b-specific antibodies in the sera of mice and guinea pigs immunized with Cap vaccine or Capfb vaccine. The D3 protein, a recombinant protein composing of nine copies of the decoy epitope (aa 169-180) of PCV2 Cap protein expressed in E. coli system (Yu et al. 2016), was used as coating antigen to detect the PCV2b decoy epitope specific antibodies in the sera of mice immunized with Capfb vaccine or Cap vaccine. The levels of specific antibody were expressed as Optical densities (OD) at $492 \mathrm{~nm} \pm$ standard deviation (SD). 


\section{Stimulation and flow cytometry assay}

ICR mice were intramuscularly immunized with $100 \mu$ of Capfb vaccine $(10 \mu \mathrm{g} /$ mouse) or $\mathrm{NaCl}$ on days 0 and 14 , stimulated with inactivated FMDV or PCV $2 \mathrm{~b}(10 \mu \mathrm{g} /$ mouse $)$ on day 21 , and sacrificed on day 22 for isolating lymph node cells on the same side as the injection site. The lymph node cells were then stained with PE-conjugated anti-CD19 and FITC-conjugated anti-CD69 antibodies for $30 \mathrm{~min}$ on ice in dark, washed with PBS, and detected by flow cytometry.

\section{Statistical analysis}

The results were analyzed using $t$ test by SPSS 19.0 software for Windows. All data were expressed as means \pm standard deviation (SD). The significance level was set at 0.05 , and $P<0.05$ was considered statistically significant.

\section{Results}

\section{Design of the PCV2b capsid protein carrying FMDV epitopes}

The neutralizing B cell epitope (aa 135-160) containing G-H loop of type O FMDV-VP1 protein was used to replace the region (aa 169-194) involving the decoy epitope and a protruding region (aa 123-151) of the PCV2b Cap protein to generate a recombinant protein designated as Capfb (Fig. 1a).

To test the effects of replacing the regions (aa 123-151 and aa 169-194) of the Cap protein with the B cell epitopes of FMDV-VP1, the secondary structure, hydrophilicity, and antigenic index of Cap and Capfb were detected with DNAStar Protean software (Fig. 1b). The secondary structures of the B cell epitopes of FMDV-VP1 in the Capfb protein (aa 123-148 and aa 166-191) and their corresponding regions in the Cap protein (aa 123-151 and aa 169-194) were similar, that all of them contained several Alpha, Beta, or Turn structures (Fig. 1b). Compared with the corresponding regions in the Cap protein, the B cell epitope of FMDV-VP1 located in positions aa $123-148$ of the Capfb protein displayed reduced hydrophilicity but increased antigenic index, while the B cell epitope of FMDV-VP1 located in aa 169-194 showed reduced hydrophilicity but similar antigenic index (Fig. 1b). These results indicated that the B cell epitopes of FMDV-VP1 in the Capfb protein showed good antigenicity and should be able to stimulate the body to produce antibodies against FMDV.

To further confirm whether the B cell epitope of FMDVVP1 in the Capfb protein could function well, the 3D structures of Capfb, Cap, and FMDV-VP1 were predicted through the Swiss Model website. We found that the 3D structure of the Cap and the Capfb protein were similar and the B cell epitopes of FMDV-VP1 in the Capfb protein regions (Fig. 1e, left panel) could exposed on the surface as their corresponding regions (aa 123-151 and aa 169-194) did in the Cap protein (Fig. 1c). Moreover, the RGD motif of the B cell epitopes of FMDV-VP1 in the Capfb protein could also form loop structure (Fig. 1e, right panel) as that in the natural FMDV-VP1 did (Fig. 1d). These results demonstrated that the B cell epitopes of FMDV-VP1 in the Capfb protein could function well and would not affect the Capfb to form VLPs.

\section{Expression, identification, and purification of Capfb}

The expression of the Capfb protein in E. coli lysates were analyzed by SDS-PAGE and Western-blot using the antiFMDV or anti-PCV2b specific antibodies as primary antibodies. The SDS-PAGE analysis showed that most of the Capfb protein with molecular weights of about $28.1 \mathrm{kDa}$ existed in the supernatant from the lysate of the $E$. coli, while little in the precipitation, indicating the Capfb were soluble proteins (Fig. 2a). The results of Western-blot revealed that the specific $28.1 \mathrm{kDa}$ band corresponding to that of the Capfb could be recognized by both the anti-FMDV and anti-PCV2b specific antibodies (Fig. 2b). Then the Capfb protein was purified by nickel affinity chromatography. The supernatant from the lysate of the E. coli was loaded onto the nickel affinity column and multiple elution fractions were obtained. The Capfb protein with high purify approximately $28.1 \mathrm{kDa}$ in the elution fractions was visible on the SDS-PAGE gel (Fig. 2c).

\section{Transmission electron microscopy analysis of Capfb}

To examine whether the Capfb protein can be assembled into VLPs, TEM analysis was performed. The results showed that the purified Capfb protein could not spontaneously form VLPs (Fig. 3a), while after being dialyzed against assembly buffer (50 mM Na2HPO4, $50 \mathrm{mM} \mathrm{NaH2PO4,} 500 \mathrm{mM} \mathrm{NaCl}$, $5 \%$ glycerol, $0.03 \%$ Tween $80,1 \%$ Triton $\mathrm{X}-100, \mathrm{pH} 6.5$ ) in vitro, the Capfb protein appeared to have assembled into particles (Fig. 3b). The assembled Cap protein was also observed under TEM as a control. The Cap protein could form homogenous and tight VLPs (Fig. 3c). In comparison, the Capfb-VLPs were looser than the Cap VLPs. The diameters of VLPs formed by both the Cap and the Capfb protein were 17-20 nm (Fig. 3b, c).

\section{Capfb-induced FMDV-specific and PCV2b-specific antibody responses in mice and guinea pigs}

To test whether the Capfb-VLPs could efficiently induce both anti-FMDV and anti-PCV2b antibodies, mice were immunized with Capfb vaccine or Cap vaccine as control, on days 0 and 14 (Fig. 4a). The levels of FMDV and PCV2b specific antibodies in the sera collected on days 0,28 , and 35 were detected by enzyme-linked immunosorbent assay (ELISA) 
Fig. 1 Design and structure prediction of Capfb. a Schematic diagram of Cap and Capfb. b Secondary structure prediction of Cap and Capfb. 3D structure prediction of $\mathbf{c}$ the Cap protein of PCV2b, $\mathbf{d}$ the VP1 protein of FMDV, and $\mathbf{d}$ the Capfb protein. Green, a protruding region (aa 123-151) of the PCV2b Cap protein. Pink, the PCV2b decoy epitope containing region (aa 169-194). Blue, the neutralizing B cell epitope (aa 135-160) of FMDV-VP1. Red, the RGD motif a

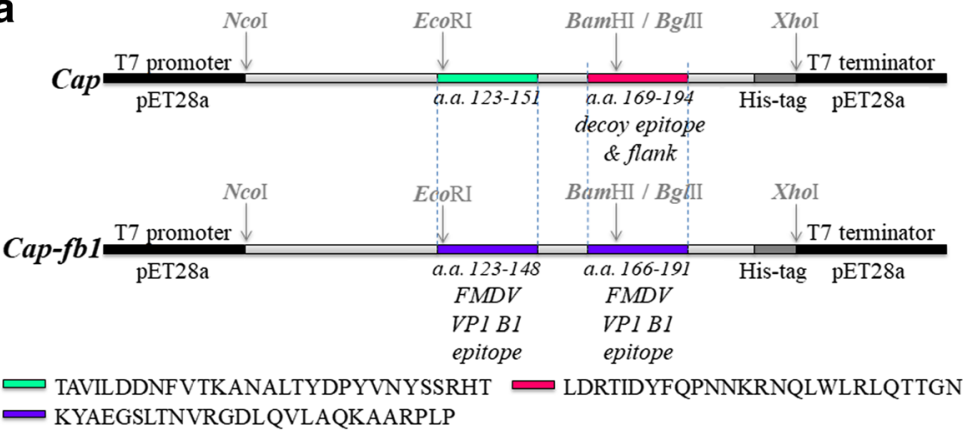

b
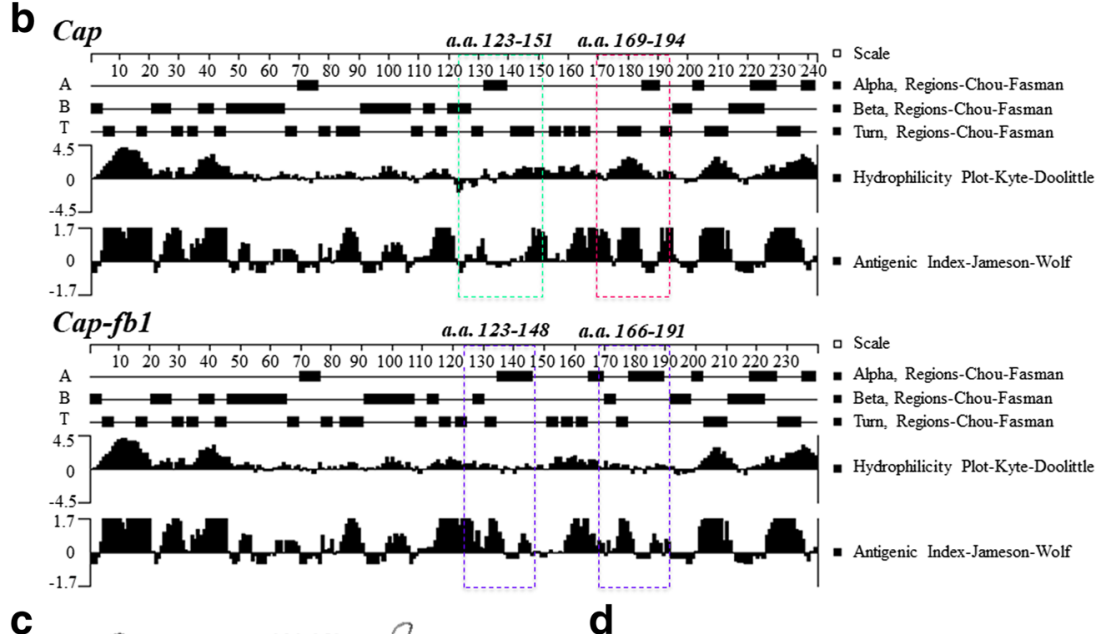

C
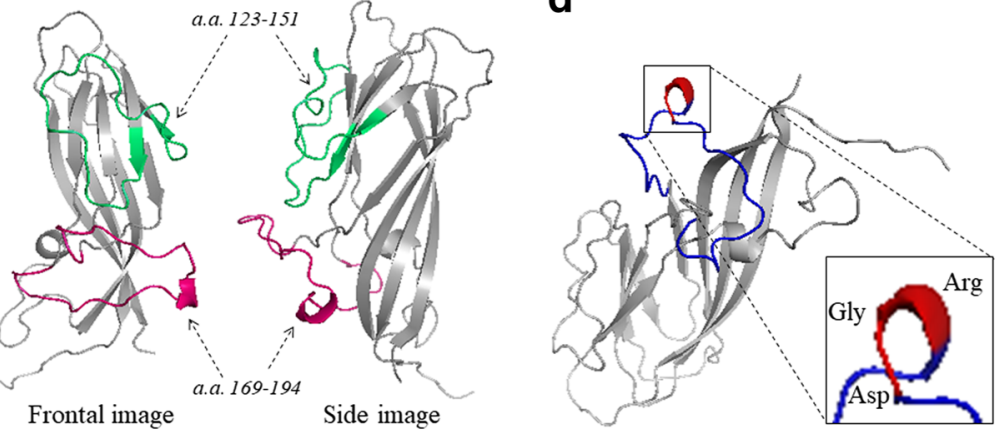

e
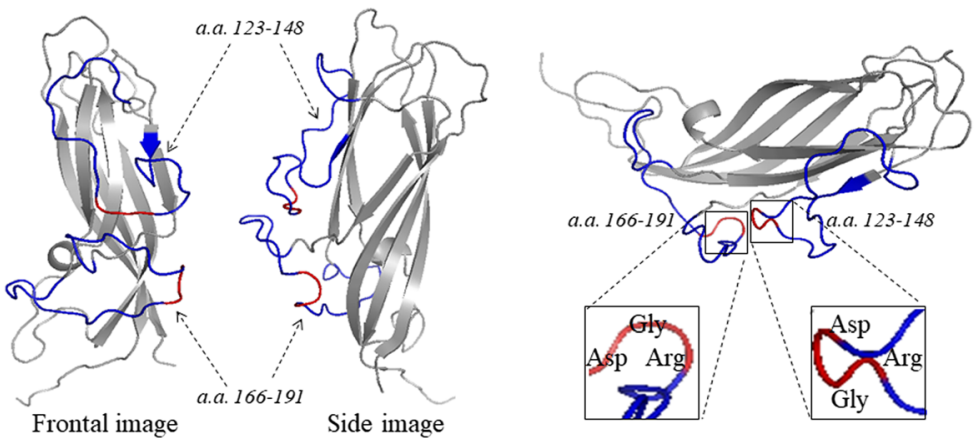

(Fig. 4a). The Capfb-VLPs could elicit vigorous antibody response against inactivated FMDV and PCV2b on days 28 and 35 (Fig. 4b), while the Cap-VLPs could only induce antiPCV2b specific IgG since it involved no B cell eptiope of FMDV-VP1 (Fig. 4c). Noticeably, the mice inoculated with the Capfb-VLPs produced significantly higher level of anti-
PCV2b specific IgG on day 35 than the mice immunized with the Cap-VLPs $(P=0.0022$, Fig. 4c). The results demonstrated that the Capfb-VLPs could induce the antibody response against inactivated FMDV and PCV2b. Moreover, the Capfb protein could stimulate the mice to produce higher level of PCV2b-specific antibodies than the Cap-VLPs. 

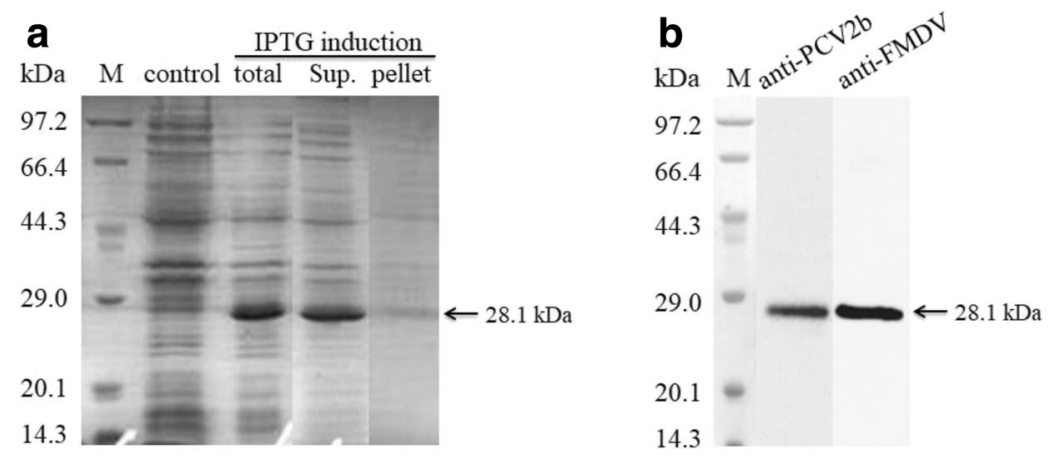

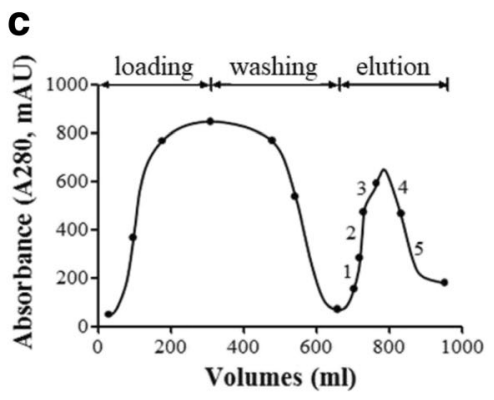

Fig. 2 Expression, identification, and purification of Capfb. a SDSPAGE analysis of Capfb expressed in E. coli. Control, lysate of E. coli without IPTG induction; total, whole cellular proteins in the lysate of E. coli; Sup., the supernatant of the lysate; pellet, the precipitation of the lysate. b Western-blot analysis of Capfb expressed in E. coli. Primary antibody is the specific antibody raised from the mice immunized with inactivated PCV2b or FMDV and secondary antibody

To further observe whether the Capfb protein could induce specific antibody response against FMDV and PCV2b in different species of animals, guinea pigs were vaccinated with Capfb vaccine on days 0,14 , and 28 and were bled on days 0 and 42 for detecting anti-FMDV IgG and anti-PCV2b IgG levels by ELISA (Fig. 4d). The results showed that the antiFMDV $\operatorname{IgG}(P=0.0305$, Fig. 4e $)$ or anti-PCV2b $\operatorname{IgG}(P=$ 0.0022 , Fig. $4 \mathrm{f}$ ) could be induced by the Capfb vaccine on days 42 , respectively. The results demonstrated that the Capfb-VLPs had the potential to be widely used for vaccination among different species of animals.

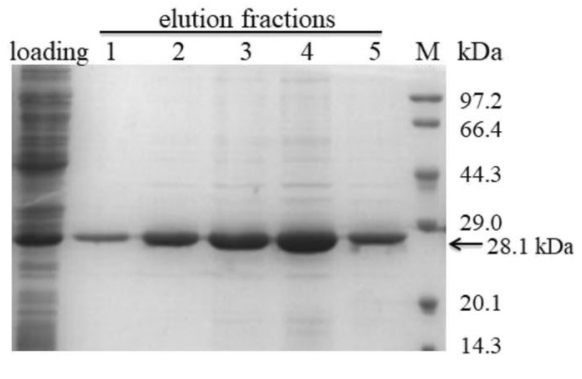

is the goat anti-mouse IgG/HRP. c SDS-PAGE analysis of purified Capfb. Loading represents the lysate of the $E$. coli loaded onto the nickel affinity column. Lanes 1-5 showed the Capfb in the elution fractions 1, 2, 3, 4, and 5 collected from the nickel affinity chromatography, respectively. M represents the molecular weight marker. The arrow denotes the Capfb with the calculated molecular weight of $28.1 \mathrm{kDa}$

\section{Decoy epitope of PCV2b-directed antibody responses in mice}

D3 protein containing nine copies of the decoy epitope of PCV2b was used as a coating antigen to detect the antidecoy epitope antibodies in the sera collected on day 28 of the mice immunized with the Capfb or Cap vaccine. As expected, anti-decoy epitope antibodies were not detected in the sera of mice immunized with the Capfb-VLPs (Fig. 5). Compared with the Capfb-VLPs, Cap-VLPs induced significantly higher level of antibodies against the decoy epitope a

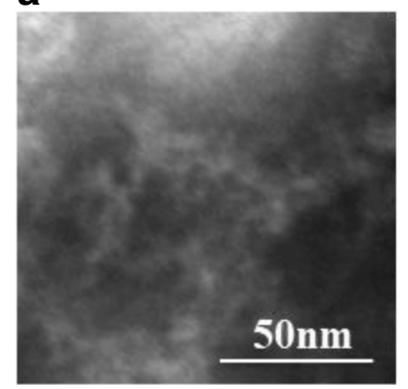

b

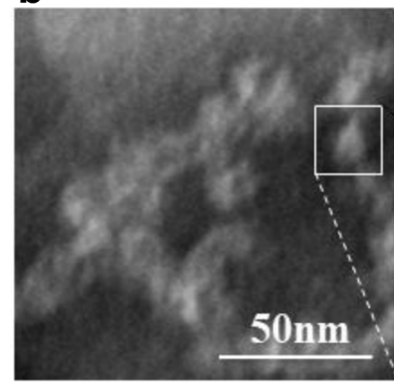

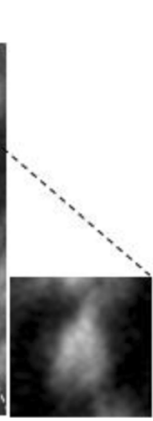

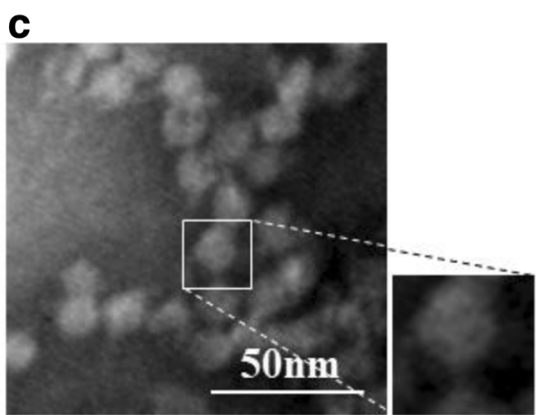

Fig. 3 Analysis of Capfb structure by transmission electron microscopy. Electron micrographs of a unassembled Capfb protein, $\mathbf{b}$ assembled Capfb VLPs, and $\mathbf{c}$ assembled Cap-VLPs at a magnification of 40,000. Bars represent $50 \mathrm{~nm}$ 
Fig. 4 Capfb-induced antibody response in mice and guinea pigs. Immunization procedure of mice (a) and guinea pigs (d). The FMDV-specific IgG was detected in the sera (1:100 dilution) of the mice (b) or guinea pigs (e) immunized with Cap or Capfb vaccine by ELISA using inactivated FMDV as coating antigens. PCV2b-specific IgG were detected in the sera (1:100 dilution) of the mice (c) or guinea pigs (f) immunized with Capfb vaccine by ELISA using inactivated PCV2b as coating antigen. Each point represents the $\mathrm{IgG}$ level of one mouse. Bars show mean $\pm \mathrm{SD}$

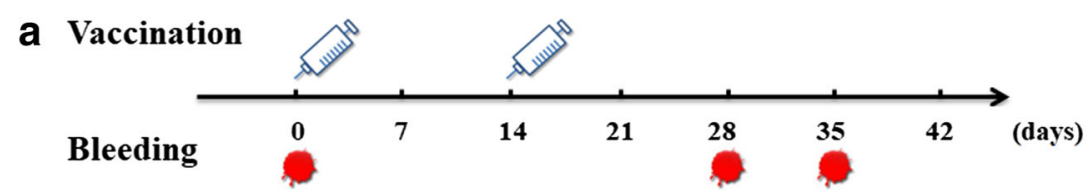

b Anti-FMDV IgG

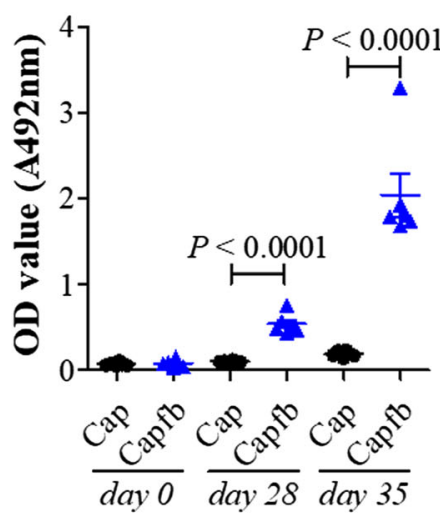

C Anti-PCV2b IgG
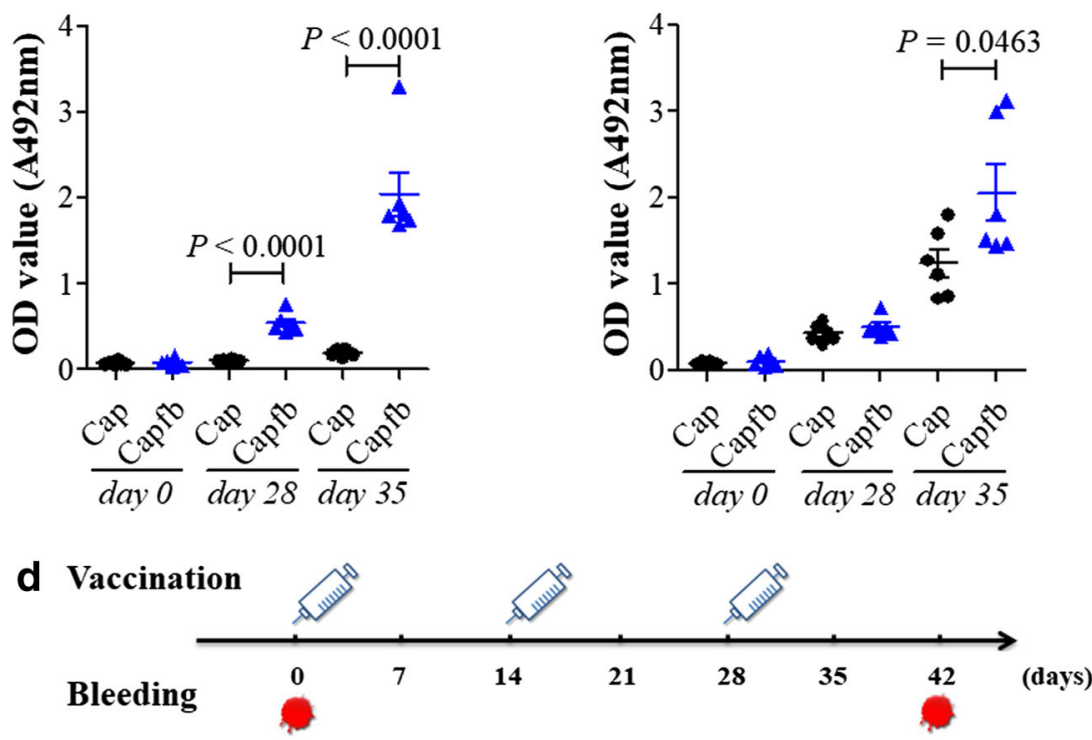

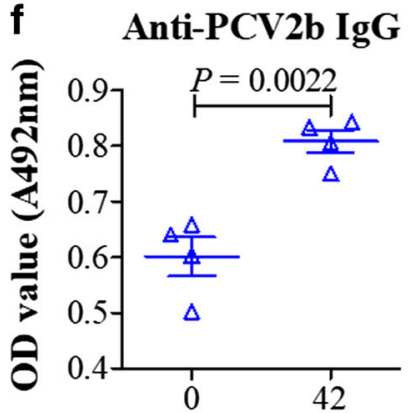

Days post immunization
( $P=0.001$, Fig. 5), indicating the decoy epitope of Cap-VLPs could be exposed during the process of the vaccination.

\section{Percentage and activation of B cells in lymph node cells of mice after stimulation by inactivated FMDV or PCV2b}

To explore whether the Capfb protein could induce immune response when mice were stimulated with FMDV or PCV2b, we immunized the mice with $\mathrm{Capfb}$ vaccine or $\mathrm{NaCl}$ as control, on days 0 and 14 , and stimulated the mice with inactivated FMDV or PCV2b on day 21 (Fig. 6a). Twentyfour hours after the stimulation, we sacrificed the mice and detected the percentage and activation of B cells in the lymph node on the same side of the injection site (Fig. 6a). The results showed that when mice were stimulated with inactivated FMDV, compared with the mice in the control group, significantly higher percentage of $\mathrm{CD} 9^{+}$cells $\left(P=0.0149\right.$, Fig. 6b) and $\mathrm{CD} 19^{+} \mathrm{CD}^{+} 9^{+}$cells $(P=$ 0.0464 , Fig. 6c) could be detected in the mice immunized with Capfb vaccine. Similarly, there were obviously higher percentage of $\mathrm{CD}^{+} 9^{+}$cells $(P=0.0326$, Fig. 6d) and $\mathrm{CD} 19^{+} \mathrm{CD}^{+} 9^{+}$cells $(P=0.0294$, Fig. $6 \mathrm{e})$ in the mice immunized with $\mathrm{Capfb}$ vaccine after stimulation by PCV2b. The results suggested that the Capfb-VLPs could enhance the percentage and activation of $B$ cells in the lymph node when mice were stimulated with inactivated FMDV or PCV2b. 
Anti-decoy epitope IgG

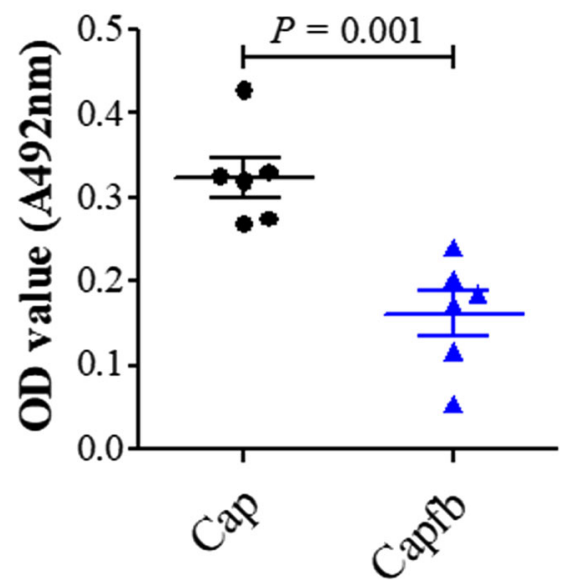

Fig. 5 Decoy epitope of PCV2b-directed antibody responses in mice. Mice were immunized with Cap or Capfb vaccine on days 0 and 14 and bled on day 28. D3 protein composing of nine copies of PCV2b decoy epitope was used as a coating antigen to detect the decoy epitope-directed IgG in the sera (1:100 dilution) of the mice by ELISA. Each point represents the IgG level of one mouse. The dotted line represents the $\mathrm{IgG}$ level in negative sera. Bars show mean $\pm \mathrm{SD}$

\section{Discussion}

To develop an effective vaccine that can protect the pigs from the mixed infection of PCV2 and FMDV, we used the neutralizing B cell epitope region (aa 135-160) to replace two regions (aa 123-151 and 169-194) of the PCV2b Cap protein to generate a recombinant protein designated as Capfb. The Capfb protein could be assembled into VLPs in vitro and induce the antibody response against both PCV2b and FMDV in mice and guinea pigs.

The VLPs generated through genetic engineering have been used as a platform carrying foreign epitopes to induce multiple immune responses (Casal 1999; Crisci et al. 2012). Chimeric PCV2-VLPs were also reported to be used as antigen vehicle carrying CSFV T cell and B cell epitopes (Zhang et al. 2014). The existing VLPs vehicle carrying foreign epitopes were expressed in baculovirus system (Casal 1999; Sedlik et al. 1997; Zhang et al. 2014). In our study, the E. coli system was used to express the Capfb, a recombinant PCV2b Cap protein carrying epitopes of FMDV-VP1. After being dialyzed in vitro, the Capfb protein could also be observed to form VLPs under TEMs. However, the VLPs formed by the Capfb seemed to be looser than that formed by the Cap protein indicating that the replacement of the two regions (aa 123-151 and 169-194) of PCV2b Cap protein with the FMDV-VP1 epitope may slightly influence the formation of the VLPs.

Though the VLPs formed by the Capfb protein did not identically mimic the natural structure of the PCV2b particles, the Capfb-VLPs still elicited vigorous anti-PCV2b antibody response in mice and guinea pigs. Noticeably, the CapfbVLPs could induce higher level of anti-PCV2b antibodies than the Cap-VLPs. The enhanced production of antiPCV $2 b$ antibodies induced by the Capfb-VLPs could be correlated with the replacement of the region (aa 169-194) containing decoy epitope. Evidences have demonstrated that the decoy epitope displayed an external loop protruding from the outer surface of the PCV2b Cap monomer, while was buried near the interface of the icosahedral PCV2 particles (Trible et al. 2012). Therefore, the decoy epitope could not induce neutralizing antibodies but diverted the humoral response away from a protective epitope (Trible et al. 2012). Incorrect fold or posttranslational modification of the recombinant protein expressed in E. coli system (Bucarey et al. 2009), may cause the exposure of the decoy epitope. In this study, the Cap-VLPs rather than the Capfb-VLPs were found to induce the decoy epitope-directed antibodies in the vaccinated mice. Thus, the replacement of the decoy epitope region in the Capfb protein could avoid the exposure of the decoy epitope and maintain the protective antibody response. Consistently, our previous study also showed that the replacement of the decoy epitope (aa 169-180) with a neutralizing epitope (aa 113-131) in the PCV2b Cap protein could increase the production of the anti-PCV2b antibodies and induces improved protective immune responses in pigs challenged with PCV2b (Yu et al. 2016).

Besides vigorous anti-PCV2b antibody response, the Capfb-VLPs could also induce high level of antibodies which could be recognized by inactivated FMDV in both mice and guinea pigs. Two regions (aa 123-151 and 169194) of the PCV2b Cap protein were chosen to insert the neutralizing B cell epitope region of FMDV-VP1, since the aa 130,131, and 169-180 were protruding elements on the surface of the PCV2b Cap protein (Saha et al. 2012; Trible et al. 2012). We tried to insert the FMDV-VP1 epitope into the aa 130-131 and aa 169-180 with or without their flanks and used DNASTA Protean software and the Swiss Model website to predict their secondary and 3D structures. We found that the FMDV-VP1 epitopes inserted into the regions aa 123-151 and aa 169-194 could protrude from the surface of the Capfb protein and mimic the natural structure, especially the RGD motif which interacts with cell surface integrin receptors and contributes to the induction of neutralizing antibodies (Jackson et al. 2002; Verdaguer et al. 1996). Therefore, we used the FMDV-VP1 epitope to replace the regions aa 123-151 and aa 169-194 of the PCV2b Cap protein. Moreover, the mice immunized with Capfb vaccine had significantly higher percentage and activation of B cells in the lymph nodes when stimulated with inactivated 
Fig. 6 Percentage and activation of B cells in lymph node cells of mice after stimulation by inactivated FMDV or PCV2b. a

Schematic diagram of

experimental procedure.

Percentage of CD19 $9^{+}$cells $(\mathbf{b}, \mathbf{d})$ and $\mathrm{CD} 19^{+} \mathrm{CD} 69^{+}$cells $(\mathbf{c}, \mathbf{e})$ in the lymph node cells on the same side of the injection site of mice (four per group) after being stimulated with inactivated FMDV or PCV2b were detected by flow cytometry assay. Bar shows mean $\pm \mathrm{SD}$ a

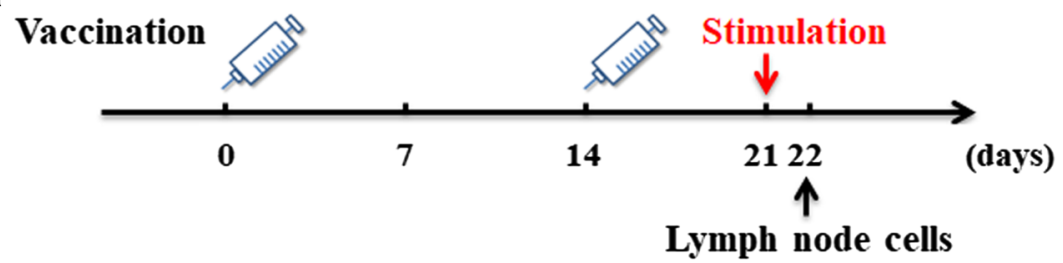

b FMDV challenge

C FMDV challenge
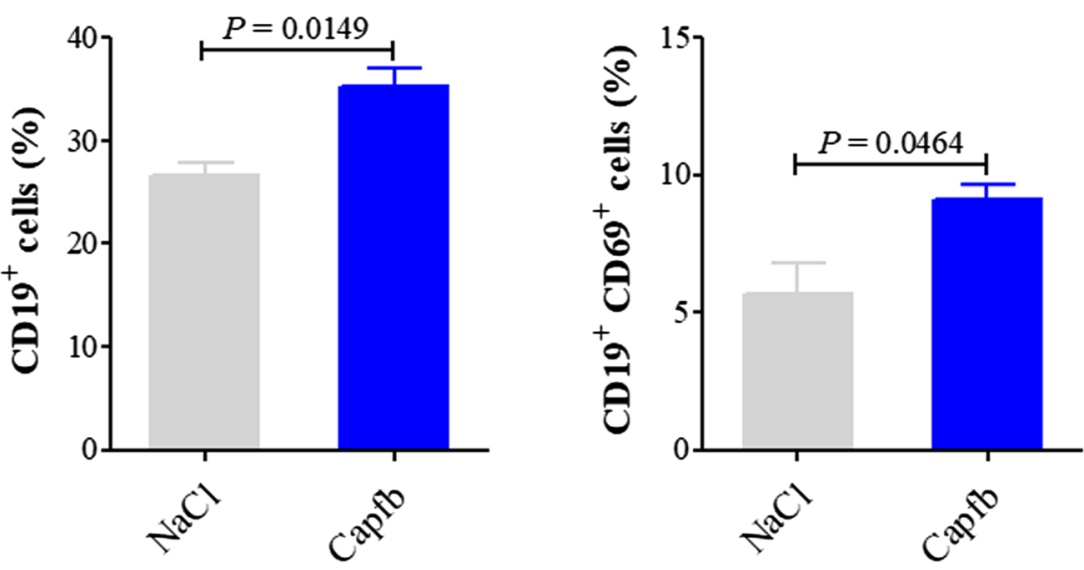

d PCV2b challenge

e PCV2b challenge

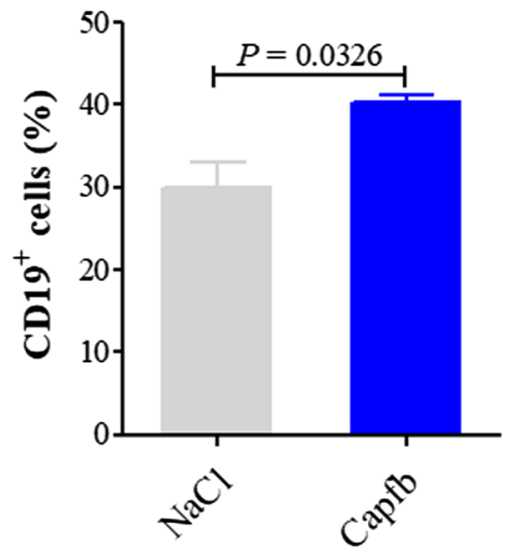

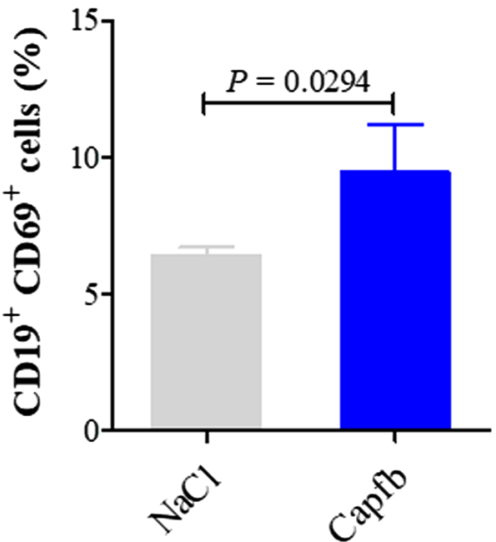

FMDV or PCV2b, indicating the Capfb protein had the potential to elicit protective immune response when animals were infected with FMDV or PCV2b. Prospectively, we may use live FMDV or PCV2b to challenge the animals immunized with the Capfb vaccine in high-level biosafety containment organisms to test the neutralizing activity of Capfb.

In the previous study, a recombinant virus PCV2 containing the epitope region (aa 141-160) of FMDV-VP1 in its Cterminal was also demonstrated to be able to induce antiPCV2-Cap protein antibodies and anti-FMDV-VP1 antibodies in mice (Huang et al. 2014). However, the recombinant virus could replicate and cause various pathological changes in mice (Huang et al. 2014); thus, the vaccines developed based on it may be infectious and have security risks. Compared with the recombinant virus, the Capfb-VLPs is not only able to elicit vigorous antibody response against PCV2b and FMDV, but also non-infectious since it did not contain viral genome.

In summary, our results suggest that the Capfb, a recombinant PCV2b Cap protein carrying FMDV-VP1 epitopes in aa 123-151 and aa 169-194 expressed in E. coli system could form VLPs through dialysis and elicit vigorous antiPCV $2 b$ and anti-FMDV antibody response in mice and guinea pigs. Thus, the Capfb-VLPs could be an efficacious candidate antigen for developing novel PCV2b-FMDV bivalent vaccine. 
Funding This study was funded by the National Nature Scientific Foundation of China (31670937 and 81471888).

\section{Compliance with ethical standards}

Conflict of interest The authors declare that they have no conflict of interests.

Ethical approval All applicable international, national, and/or institutional guidelines for the care and use of animals were followed.

\section{References}

Andersen MH, Schrama D, Straten PT, Becker JC (2006) Cytotoxic T cells. J Invest Dermatol 126(1):32-41. https://doi.org/10.1038/sj.jid. 5700001

Alexandersen S, Zhang Z, Donaldson AI, Garland AJ (2003) The pathogenesis and diagnosis of foot-and-mouth disease. J Comp Pathol 129(1):1-36

Allan GM, McNeilly F, Ellis J, Krakowka S, Botner A, McCullough K, Nauwynck H, Kennedy S, Meehan B, Charreyre C (2004) PMWS: experimental model and co-infections. Vet Microbiol 98(2):165-168

Beach NM, Meng XJ (2012) Efficacy and future prospects of commercially available and experimental vaccines against porcine circovirus type 2 (PCV2). Virus Res 164(1-2):33-42. https://doi. org/10.1016/j.virusres.2011.09.041

Bittle JL, Houghten RA, Alexander H, Shinnick TM, Sutcliffe JG, Lerner RA, Rowlands DJ, Brown F (1982) Protection against foot-and-mouth disease by immunization with a chemically synthesized peptide predicted from the viral nucleotide sequence. Nature 298(5869):30-33

Bucarey SA, Noriega J, Reyes P, Tapia C, Saenz L, Zuniga A, Tobar JA (2009) The optimized capsid gene of porcine circovirus type 2 expressed in yeast forms virus-like particles and elicits antibody responses in mice fed with recombinant yeast extracts. Vaccine 27(42):5781-5790. https://doi.org/10.1016/j.vaccine.2009.07.061

Casal JI (1999) Use of parvovirus-like particles for vaccination and induction of multiple immune responses. Biotechnol Appl Biochem 29(Pt 2):141-150

Crisci E, Barcena J, Montoya M (2012) Virus-like particles: the new frontier of vaccines for animal viral infections. Vet Immunol Immunopathol 148(3-4):211-225. https://doi.org/10.1016/j. vetimm.2012.04.026

Doel TR, Collen T (1982) Qualitative assessment of 146 S particles of foot-and-mouth disease virus in preparations destined for vaccines. $\mathrm{J}$ Biol Stand 10(2):69-81

Franzo G, Cortey M, Segales J, Hughes J, Drigo M (2016) Phylodynamic analysis of porcine circovirus type 2 reveals global waves of emerging genotypes and the circulation of recombinant forms. Mol Phylogenet Evol 100:269-280. https://doi.org/10.1016/j.ympev. 2016.04.028

Gillespie J, Opriessnig T, Meng XJ, Pelzer K, Buechner-Maxwell V (2009) Porcine circovirus type 2 and porcine circovirus-associated disease. J Vet Intern Med 23(6):1151-1163. https://doi.org/10.1111/ j.1939-1676.2009.0389.x

Grubman MJ, Baxt B (2004) Foot-and-mouth disease. Clin Microbiol Rev 17(2):465-493

Huang L, Zhang F, Tang Q, Wei Y, Wu H, Guo L, Fu Y, Liu C (2014) A recombinant porcine circovirus type 2 expressing the VP1 epitope of the type $O$ foot-and-mouth disease virus is infectious and induce both PCV2 and VP1 epitope antibodies. Appl Microbiol Biotechnol 98(22): 9339-9350. https://doi.org/10.1007/s00253-014-5994-y

Huang LP, Lu YH, Wei YW, Guo LJ, Liu CM (2011) Identification of one critical amino acid that determines a conformational neutralizing epitope in the capsid protein of porcine circovirus type 2. BMC Microbiol 11:188. https://doi.org/10.1186/1471-2180-11-188

Jackson T, Mould AP, Sheppard D, King AM (2002) Integrin alphavbeta1 is a receptor for foot-and-mouth disease virus. J Virol 76(3):935-941

Kupper H, Keller W, Kurz C, Forss S, Schaller H, Franze R, Strohmaier K, Marquardt O, Zaslavsky VG, Hofschneider PH (1981) Cloning of cDNA of major antigen of foot and mouth disease virus and expression in E. coli. Nature 289(5798):555-559

Mankertz A, Caliskan R, Hattermann K, Hillenbrand B, Kurzendoerfer P, Mueller B, Schmitt C, Steinfeldt T, Finsterbusch T (2004) Molecular biology of Porcine circovirus: analyses of gene expression and viral replication. Vet Microbiol 98(2):81-88

Nawagitgul P, Morozov I, Bolin SR, Harms PA, Sorden SD, Paul PS (2000) Open reading frame 2 of porcine circovirus type 2 encodes a major capsid protein. J Gen Virol 81(Pt 9):2281-2287. https://doi. org/10.1099/0022-1317-81-9-2281

Opriessnig T, Meng XJ, Halbur PG (2007) Porcine circovirus type 2 associated disease: update on current terminology, clinical manifestations, pathogenesis, diagnosis, and intervention strategies. J Vet Diagn Investig 19(6):591-615. https://doi.org/10.1177/ 104063870701900601

Pfaff E, Mussgay M, Bohm HO, Schulz GE, Schaller H (1982) Antibodies against a preselected peptide recognize and neutralize foot and mouth disease virus. EMBO J 1(7):869-874

Saha D, Huang L, Bussalleu E, Lefebvre DJ, Fort M, Van Doorsselaere J, Nauwynck HJ (2012) Antigenic subtyping and epitopes' competition analysis of porcine circovirus type 2 using monoclonal antibodies. Vet Microbiol 157(1-2):13-22. https://doi.org/10.1016/j. vetmic.2011.11.030

Saiz M, Nunez JI, Jimenez-Clavero MA, Baranowski E, Sobrino F (2002) Foot-and-mouth disease virus: biology and prospects for disease control. Microbes Infect 4(11):1183-1192

Sedlik C, Saron M, Sarraseca J, Casal I, Leclerc C (1997) Recombinant parvovirus-like particles as an antigen carrier: a novel nonreplicative exogenous antigen to elicit protective antiviral cytotoxic $\mathrm{T}$ cells. Proc Natl Acad Sci U S A 94(14):7503-7508

Shao JJ, Wong CK, Lin T, Lee SK, Cong GZ, Sin FW, Du JZ, Gao SD, Liu XT, Cai XP, Xie Y, Chang HY, Liu JX (2011) Promising multiple-epitope recombinant vaccine against foot-and-mouth disease virus type $O$ in swine. Clin Vaccine Immunol 18(1):143-149. https://doi.org/10.1128/CVI.00236-10

Stenfeldt C, Pacheco JM, Rodriguez LL, Arzt J (2014) Infection dynamics of foot-and-mouth disease virus in pigs using two novel simulated-natural inoculation methods. Res Vet Sci 96(2):396405. https://doi.org/10.1016/j.rvsc.2014.01.009

Trible BR, Suddith AW, Kerrigan MA, Cino-Ozuna AG, Hesse RA, Rowland RR (2012) Recognition of the different structural forms of the capsid protein determines the outcome following infection with porcine circovirus type 2. J Virol 86(24):13508-13514. https://doi.org/10.1128/JVI.01763-12

Verdaguer N, Mateu MG, Bravo J, Domingo E, Fita I (1996) Induced pocket to accommodate the cell attachment Arg-Gly-Asp motif in a neutralizing antibody against foot-and-mouth-disease virus. J Mol Biol 256(2):364-376. https://doi.org/10.1006/jmbi.1996.0092

Yu C, Li X, Liu J, Diao W, Zhang L, Xiao Y, Wei H, Yu Y, Yu Y, Wang L (2016) Replacing the decoy epitope of $P C V 2 b$ capsid protein with a protective epitope enhances efficacy of $P C V 2 b$ vaccine. Vaccine 34(50):6358-6366. https://doi.org/10.1016/j.vaccine.2016.10.044

Zeltins A (2013) Construction and characterization of virus-like particles: a review. Mol Biotechnol 53(1):92-107. https://doi.org/10.1007/ s12033-012-9598-4

Zhang H, Qian P, Liu L, Qian S, Chen H, Li X (2014) Virus-like particles of chimeric recombinant porcine circovirus type 2 as antigen vehicle carrying foreign epitopes. Viruses 6(12):4839-4855. https://doi.org/ $10.3390 / \mathrm{v} 6124839$ 